\section{(6) OPEN ACCESS}

\title{
Outcomes in UK patients with hospital-acquired bacteraemia and the risk of catheter-associated urinary tract infections
}

\author{
Mark Melzer, ${ }^{1}$ Catherine Welch ${ }^{2}$
}

${ }^{1}$ Department of Infection, Barts Health NHS Trust, Royal London Hospital, London, UK ${ }^{2}$ Department of Primary Care and Population Health, University College London, London, UK

\section{Correspondence to} Dr Mark Melzer, Department of Infection, Barts Health NHS Trust, Royal London Hospital, 3rd floor Pathology and Pharmacy Building, 80 Newark Street, London E1 2ES, UK; mark.melzer@bartshealth.nhs. uk

Received 15 August 2012 Revised 23 January 2013 Accepted 24 February 2013 Published Online First 21 March 2013

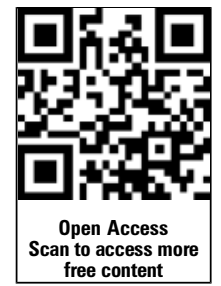

To cite: Melzer M Welch C. Postgrad Med J 2013;89:329-334.

\begin{abstract}
Purpose There is lack of contemporary outcome data on patients with hospital-acquired infections that cause bacteraemia. We determined the risk factors for 7-day mortality and investigated the hypothesis that, compared with central venous catheter (CVC)-associated bacteraemic infections, catheter-associated bacteraemic urinary tract infections (UTIs) were significantly associated with 7-day mortality.

Methods From October 2007 to September 2008, demographical, clinical and microbiological data were collected on patients with hospital-acquired bacteraemia. Patients were followed until death, hospital discharge or recovery from infection. Risk factors for 7-day mortality were determined and multivariate logistic regression was used to define the association between catheter-associated bacteraemic UTIs and likelihood of death.
\end{abstract}

Results 559 bacteraemic episodes occurred in 437 patients. Overall, there were 90 deaths (20.6\%) at 7 days and 153 deaths $(35.0 \%)$ at 30 days. Among patients with catheter-associated bacteraemic UTIs, 7-day and 30day mortalities associated with each bacteraemic episode were 25/83 (30.1\%) and 33/83 (39.8\%), respectively. Within this subgroup, the commonest isolates were Escherichia coli, $36(43.4 \%)$, Proteus mirabilis, 11 $(13.3 \%)$ and Pseudomonas aeruginosa, 9 (10.8\%). There were 22 (26.5\%) multiple drug-resistant isolates and, of the $E$ coli infections, 6 (16.7\%) were extended spectrum $\beta$-lactamase producers. In univariate analysis, the variables found to have the strongest association with 7-day mortality were age, Pitt score, Charlson comorbidity index (CCI), medical speciality and site of infection. Compared with CVC-associated bacteraemic infections, there was a significant association between catheterassociated bacteraemic UTIs and 7-day mortality (OR $4.16,95 \% \mathrm{Cl} 1.86$ to 9.33 ). After adjustment for age and $\mathrm{CCl}$, this association remained significant (OR 2.90, 95\% Cl 1.19 to 7.07 ).

Conclusions Compared with CVC-associated bacteraemic infections, catheter-associated bacteraemic UTIs were significantly associated with 7-day mortality. Efforts to reduce these infections should be prioritised.

\section{INTRODUCTION}

Hospital-acquired bacteraemia is a significant cause of patient mortality and morbidity. ${ }^{1}$ Sites of infection causing hospital-acquired bacteraemia are well described and commonly include medical devices such as central venous catheters (CVCs) and urinary catheters. ${ }^{2}$ In consecutive patients with hospital-acquired bacteraemia, the relative contribution of these sites to 7-day mortality has not been previously described. Since the introduction of mandatory meticillin-resistant Staphylococcus aureus (MRSA) bacteraemia in 2002, and its use as a key quality indicator in UK National Health Service trusts, attention has primarily focused on reducing intravascular catheter-associated infections rather than catheter-associated urinary tract infections (UTIs).

Worldwide, catheter-associated UTIs result from the widespread use of urinary catheters in hospitals, much of which is inappropriate. ${ }^{3-5}$ Efforts to reduce these infections have been made by limiting catheter insertion to patients with urinary outflow obstruction, patients undergoing surgical procedures or patients requiring urinary output monitoring as part of medical management. ${ }^{6}$ Standard aseptic techniques for catheter insertion are well described, ${ }^{7}$ and timely removal of catheter is an important factor in reducing infection. ${ }^{8}$ International guidance has been published on the prevention and management of catheter-associated UTIs, most recently from the Infectious Diseases Society of America. ${ }^{9}$ Despite their widely recognised importance as a significant cause of hospital-acquired infection, there is lack of contemporary outcome data on patients with bacteraemic episodes caused by catheter-associated UTIs.

The aims of this study were to determine the risk factors for 7-day mortality in consecutive patients with hospital-acquired bacteraemia. We hypothesised that compared with CVC-associated bacteraemic infection, catheter-associated bacteraemic UTIs were more likely to cause death within 7 days.

\section{METHODS}

\section{Study setting}

The study was undertaken at Barking, Havering and Redbridge University Hospitals NHS Trust (BHRUT). This is composed of Queen's Hospital with a 900-bedded major acute hospital and King George Hospital with a 600-bedded local district hospital. BHRUT serves an elderly and ethnically diverse population of 750000 patients in Essex, adjacent to East London. In addition to general surgery, medicine, paediatric and maternity services, BHRUT has 30 high-dependency and critical care beds (including neurosurgical beds), a specialist ward for haematology and oncology patients and a high-level neonatal intensive care unit.

\section{Study population}

From October 2007 to September 2008, consecutive in-patients with hospital-acquired bacteraemia were prospectively studied. 


\section{Definitions}

Significant bacteraemia was defined as a blood culture isolate, unlikely to be an environmental or skin contaminant, ascertained from a patient with a compatible clinical syndrome. This decision was based on the patient's history, examination findings, response to antimicrobial treatment, blood culture isolate and bacterial cultures from other body sites. Hospital-acquired bacteraemia was defined as a positive blood culture obtained at least $48 \mathrm{~h}$ after hospital admission. Specialities at the time of treatment of bacteraemia were medicine, surgery, critical care, obstetrics and gynaecology and paediatrics. Resistance profiles were classified as sensitive, partial or multiple drug resistance (MDR). Partial resistance was defined as the resistance to at least one antibiotic class, and MDR as the resistance to three or more antibiotic classes that were commonly used to treat a specific bacterial isolate.

Sites of infection were defined according to the Centers for Disease Control and Prevention surveillance definitions. ${ }^{10}$ For CVC-associated infection, this was defined as evidence of infection (erythema, induration or pus) at a CVC exit site or isolation of the same organism from the blood or line tip. Catheter-associated UTIs were defined as infection in patients with indwelling urethral or suprapubic catheters, or patients who intermittently self-catheterised, in the presence of symptoms or signs compatible with a UTI where no other source was identified. Bacteraemias in patients with an unknown source were classified as undefined. Subsequent bacteraemic episodes were defined as an infection with the same organism occurring more than 28 days after the first episode, or a bacteraemic episode with a different organism occurring within 28 days.

Appropriate treatment was defined as any component of an antibiotic regimen, empiric or definitive, used to treat an infection to which the organism was susceptible in vitro'. ${ }^{11}$ Delay in treatment was measured from the time a significant blood culture was obtained from the patient. In all patients, this was irrespective of the onset of sepsis and, in catheterised patients, the duration of urinary catheterisation.

\section{Data collection}

Attending physicians were encouraged to obtain blood cultures from patients presenting with symptoms and signs suggestive of sepsis before administration of empirical antibiotics. ${ }^{12}$ Demographical, laboratory and clinical data were collected on all in-patients who developed bacteraemia more than $48 \mathrm{~h}$ after admission. Five consultant microbiologists, separately covering five different areas making individual judgements, reviewed the case notes within $72 \mathrm{~h}$ of laboratory confirmation. In addition to demographical data, speciality at the time of bacteraemia, site of infection, organism, susceptibility profile, delay in appropriate treatment, Pitt bacteraemia score, Charlson comorbidity index (CCI) score ${ }^{13}$ and outcomes were recorded. The Pitt bacteraemia score is a validated index used in other studies to quantify severity of infection, ${ }^{14}$ based on mental status, need for ventilation and vital signs. Patients were followed up until death, discharge home or to a rehabilitation ward or recovery from infection.

Patients were treated empirically according to the local guidelines. Once susceptibility profiles were known, antibiotic treatment regimens were rationalised to narrow spectrum agents. All-cause outcomes were recorded as survived or died at 7 and 30 days. It was assumed that patients who were discharged prior to 7 or 30 days survived until 7 or 30 days.
Table 1 Demographical details, Charlson comorbidity index and Pitt bacteraemia score of 437 consecutive patients with 559 hospital-acquired bacteraemic episodes

\begin{tabular}{|c|c|c|c|c|}
\hline & \multicolumn{2}{|c|}{ 7-day mortality } & \multicolumn{2}{|c|}{ 30-day mortality } \\
\hline & Survived (\%) & Died (\%) & Survived (\%) & Died (\%) \\
\hline Total & $347(79.4)$ & $90(20.6)$ & $284(65.0)$ & $153(35.0)$ \\
\hline \multicolumn{5}{|l|}{ Age (years) } \\
\hline$<1$ & $27(100.0)$ & $0(0.0)$ & $27(100.0)$ & $0(0.0)$ \\
\hline $1-15$ & $7(100.0)$ & $0(0.0)$ & $7(100.0)$ & $0(0.0)$ \\
\hline $16-30$ & $12(85.7)$ & $2(14.3)$ & $11(78.6)$ & $3(21.4)$ \\
\hline $31-50$ & $36(92.3)$ & $3(7.7)$ & $33(84.6)$ & $6(15.4)$ \\
\hline $51-70$ & 99 (83.9) & $19(16.1)$ & $80(67.8)$ & $38(32.2)$ \\
\hline$>70$ & 166 (71.6) & $66(28.4)$ & $126(54.3)$ & $106(45.7)$ \\
\hline \multicolumn{5}{|l|}{ Sex } \\
\hline Male & $187(80.6)$ & 45 (19.4) & $156(67.2)$ & $76(32.8)$ \\
\hline Female & $160(78.0)$ & $45(22.0)$ & $128(62.4)$ & $77(37.6)$ \\
\hline \multicolumn{5}{|l|}{ Site $^{*}$} \\
\hline Queen's Hospital & $283(82.0)$ & $62(18.0)$ & $237(68.7)$ & $108(31.3)$ \\
\hline King George Hospital & $169(79.0)$ & $45(21.0)$ & $135(63.1)$ & 79 (36.9) \\
\hline \multicolumn{5}{|l|}{ Speciality* } \\
\hline Critical care & $134(82.2)$ & $29(17.8)$ & $109(66.9)$ & $54(33.1)$ \\
\hline Medicine & $185(74.3)$ & $64(25.7)$ & $144(57.8)$ & $105(42.2)$ \\
\hline Surgical & $72(83.7)$ & $14(16.3)$ & $61(70.9)$ & $25(29.1)$ \\
\hline $\begin{array}{l}\text { Obstetrics and } \\
\text { gynaecology }\end{array}$ & $6(100.0)$ & $0(0.0)$ & $6(100.0)$ & $0(0.0)$ \\
\hline $\begin{array}{l}\text { Paediatrics and } \\
\text { neonatology }\end{array}$ & $40(100.0)$ & $0(0.0)$ & $40(100.0)$ & $0(0.0)$ \\
\hline Othert & $15(100.0)$ & $0(0.0)$ & $12(80.0)$ & $3(20.0)$ \\
\hline Neutropenia* & $27(79.4)$ & $7(20.6)$ & $24(70.6)$ & $10(29.4)$ \\
\hline \multicolumn{5}{|l|}{ Pitt score* } \\
\hline 0 & $90(92.8)$ & $7(7.2)$ & $80(82.5)$ & $17(17.5)$ \\
\hline 1 & 85 (87.6) & $12(12.4)$ & 76 (78.4) & $21(21.6)$ \\
\hline 2 & 85 (88.5) & $11(11.5)$ & 76 (79.2) & $20(20.8)$ \\
\hline 3 & $43(74.1)$ & $15(25.9)$ & $32(55.2)$ & $26(44.8)$ \\
\hline 4 & $63(74.1)$ & $22(25.9)$ & $40(47.1)$ & $45(52.9)$ \\
\hline $5-7$ & $62(72.1)$ & $24(27.9)$ & $47(54.7)$ & $39(45.3)$ \\
\hline $8-9$ & $6(31.6)$ & $13(68.4)$ & $4(21.1)$ & $15(78.9)$ \\
\hline \multicolumn{5}{|l|}{$\mathrm{CCl}^{*}$} \\
\hline 0 & 68 (93.2) & $5(6.8)$ & $66(90.4)$ & 7 (9.6) \\
\hline 1 & $84(87.5)$ & $12(12.5)$ & $68(70.8)$ & $28(29.2)$ \\
\hline 2 & 90 (82.6) & $19(17.4)$ & $75(68.8)$ & $34(31.2)$ \\
\hline 3 & $74(78.7)$ & $20(21.3)$ & $65(69.1)$ & $29(30.9)$ \\
\hline 4 & 37 (72.5) & $14(27.5)$ & $27(52.9)$ & $24(47.1)$ \\
\hline 5 & $30(81.1)$ & 7 (18.9) & $21(56.8)$ & $16(43.2)$ \\
\hline 6 & 31 (70.5) & $13(29.5)$ & $25(56.8)$ & $19(43.2)$ \\
\hline $7-9$ & 31 (64.6) & 17 (35.4) & 19 (39.6) & $29(60.4)$ \\
\hline \multicolumn{5}{|l|}{ Site of infection* } \\
\hline CVC & $110(92.4)$ & $9(7.6)$ & $92(77.3)$ & $27(22.7)$ \\
\hline Catheter-associated UTI & $58(69.9)$ & $25(30.1)$ & $50(60.2)$ & $33(39.8)$ \\
\hline $\begin{array}{l}\text { Non-catheter-associated } \\
\text { UTI }\end{array}$ & $45(77.6)$ & $13(22.4)$ & $39(67.2)$ & $19(32.8)$ \\
\hline Biliary tract & $13(92.9)$ & $1(7.1)$ & 10 (71.4) & $4(28.6)$ \\
\hline Other & $225(79.2)$ & $59(20.8)$ & $180(63.4)$ & 104 (36.6) \\
\hline
\end{tabular}

\section{Microbiology data}

Blood cultures were analysed using an automated system BacT/ ALERT3D (bioMérieux, Mary l'Etoile, France). Gram-negative organisms were speciated by biochemical tests using api20E and 
Figure 1 Sites of infection in consecutive patients with hospital-acquired bacteraemia, expressed as percentages. This figure is only reproduced in colour in the online version.

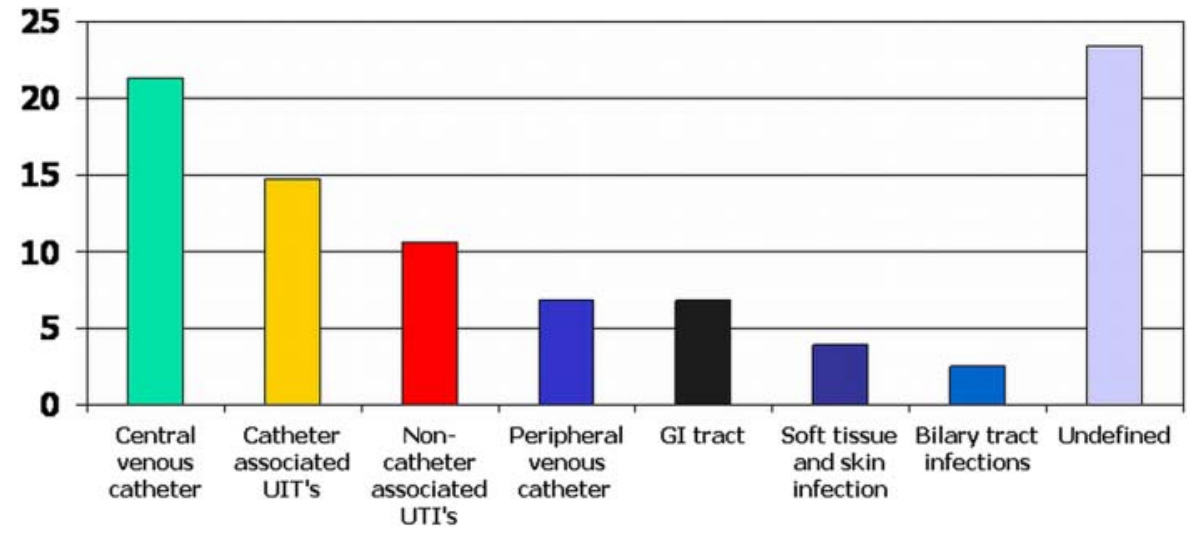

api20NE (bioMérieux). Antibiotic susceptibility testing was performed by standardised British Society of Antimicrobial Chemotherapy methods. ${ }^{15}$

\section{Data analysis}

Quantitative data are presented as numbers with percentages.

Univariate analysis was used to examine the association between predictor variables and likelihood of death at 7 days. The main outcome of interest was the association with catheterassociated UTIs. For sites of infection, CVC infection was used as the baseline category when performing regression analysis. This was chosen because CVC-associated infections are welldefined and are associated with a large number of bacteraemic episodes. Other variables investigated were age, gender, speciality at the time of bacteraemia, Pitt score, CCI, neutropenia, delay in appropriate treatment (measured in days) and organism susceptibility. If a patient was presented with more than one bacteraemic episode caused by the same organism within 28 days, it was assumed that the final episode had the strongest association with mortality so only this episode was analysed.

Age and CCI confound the association between catheterassociated UTI and death. Therefore, these variables were selected a priori to be included in multivariate logistic regression used to examine the association between site of infection and death at 7 days. Prior to fitting this model, bacteraemic episodes were excluded if the CCI was missing. Generalised estimating equations were used to account for dependency between multiple bacteraemic episodes for patients in the univariate and multivariate analysis. Data were analysed using Stata SE (V.11.1).

\section{Clinical governance}

The clinical governance committee of BHRUT approved this study. Ethical approval was not required.

Figure 2 Proportion of bacteraemic isolates causing catheter-associated urinary tract infections E. coli, Escherichia coli; P. mirabilis, Proteus mirabilis; P. aeruginosa, Pseudomonas aeruginosa; K. pneumoniae, Klebsiella pneumoniae; E. faecalis, Enterococcus faecalis; C. koseri, Citrobacter koseri; E. cloacae, Enterobacter cloacae; M. morganii, Morganella morganii; S. liquefacians, Serratia liquefacians; P. stuartii; Providencia stuartii; K. oxytoca, Klebsiella oxytoca; S. marcescens, Serratia marcescens; $S$. salivarus, Streptococcus salivarus. This figure is only reproduced in colour in the online version.

\section{RESULTS}

There were 559 episodes of bacteraemia in 437 patients with bacteraemic hospital-acquired infections. Eighty-four patients had multiple bacteraemic episodes associated with the same sites of infection. A total of $232(53.1 \%)$ patients were aged 70 or more. Two hundred and thirty-two patients (53.1\%) were men. Most bacteraemic episodes occurred on medical (249 (44.5\%)) and critical care wards (163 (29.2\%)), and at 7 and 30 days most deaths occurred in medical patients compared with other specialities (table 1). After CVC-associated bacteraemic infection, the second commonest site was catheter-associated bacteraemic UTI (70 patients with 83 (14.8\%) bacteraemic episodes) figure 1 . Fifty-six $(80 \%)$ patients with catheter-associated bacteraemic UTI were older than 70 years. There were $30(36.1 \%)$ bacteraemic episodes in patients with catheter-associated bacteraemic UTI and a CCI $>3$, fewer than bacteraemic episodes associated with biliary tract infection $7 / 14(50.0 \%)$ but a similar or greater proportion to other sites of infection.

Overall, there were 90 (20.6\%) deaths within 7 days and 153 (35.0\%) within 30 days following a bacteraemic episode (table 1). Seven-day and 30-day mortalities associated with a bacteraemic episode caused by catheter-associated UTIs were 25/83 (30.1\%) and 33/83 (39.8\%), respectively. Among bacteraemic patients with catheter-associated UTIs, 77 (92.8\%) isolates were Gram negative and $6(7.2 \%)$ isolates were Gram positive. The three commonest bacteraemic isolates were Escherichia coli, 36 (43.4\%), Proteus mirabilis, 11 (13.3\%) and Pseudomonas aeruginosa, 9 (10.8\%). Other isolates are shown in figure 2. There were 22 (26.5\%) MDR isolates and, of the E coli infections, 6 (16.7\%) were extended spectrum $\beta$-lactamase producers. No urine samples were received or processed from $15(18.1 \%)$ of the 83 patients with catheterassociated bacteraemic UTIs. Of the samples received, 35 (42.2\%) grew mixed organisms including coliforms, 19 (22.9\%) grew the 
same organism, 7 (8.4\%) grew a different organism and 7 (8.4\%) demonstrated sterile pyuria.

Univariate analyses of mortality at 7 days are presented in table 2. The variables found to have the strongest association with 7-day mortality were age, Pitt score, CCI, medical speciality and site of infection. Compared with CVC-associated bacteraemic infections, there was a significant association between catheter-associated UTIs and 7-day mortality (OR 4.16, 95\% CI 1.86 to 9.33 ). The results of multivariate logistic regression analysis are presented in table 3. About 434 patients with 552

Table 2 Univariate analysis of mortality at 7-days

\begin{tabular}{|c|c|c|}
\hline & Crude OR $(95 \% \mathrm{Cl})$ & p Value \\
\hline \multicolumn{3}{|l|}{ Age (years) } \\
\hline$<31$ & 1 & \\
\hline $31-50$ & $2.19(0.37$ to 13.15$)$ & 0.389 \\
\hline $51-70$ & 4.89 (1.11 to 21.54$)$ & 0.036 \\
\hline$>70$ & 9.71 (2.30 to 40.97$)$ & 0.002 \\
\hline \multicolumn{3}{|l|}{ Gender } \\
\hline Female & 1 & \\
\hline Male & 0.90 (0.58 to 1.42$)$ & 0.662 \\
\hline \multicolumn{3}{|c|}{ Speciality at the time of first episode } \\
\hline Critical care & 1 & \\
\hline Medical & 1.93 (1.14 to 3.27$)$ & 0.014 \\
\hline Other & $0.55(0.27$ to 1.10$)$ & 0.093 \\
\hline Neutropoenia & $1.26(0.52$ to 3.02$)$ & 0.609 \\
\hline \multicolumn{3}{|l|}{ Pitt score } \\
\hline 0 & 1 & \\
\hline 1 & $1.92(0.75$ to 4.92$)$ & 0.175 \\
\hline 2 & 1.63 (0.62 to 4.27$)$ & 0.323 \\
\hline 3 & 4.08 (1.58 to 10.56$)$ & 0.004 \\
\hline 4 & 4.01 (1.63 to 9.86$)$ & 0.002 \\
\hline $5-7$ & 4.69 (1.94 to 11.35$)$ & 0.001 \\
\hline $8-9$ & 22.44 (6.53 to 77.11$)$ & $<0.001$ \\
\hline \multicolumn{3}{|l|}{$\mathrm{CCl}$} \\
\hline 0 & 1 & \\
\hline 1 & $1.77(0.62$ to 5.05$)$ & 0.285 \\
\hline 2 & 2.95 (1.10 to 7.87$)$ & 0.031 \\
\hline 3 & $3.17(1.18$ to 8.55$)$ & 0.023 \\
\hline 4 & $4.50(1.56$ to 13.01$)$ & 0.005 \\
\hline 5 & 2.15 (0.63 to 7.39$)$ & 0.224 \\
\hline 6 & 4.70 (1.58 to 13.98$)$ & 0.005 \\
\hline $7-9$ & 5.77 (2.00 to 16.61$)$ & 0.001 \\
\hline \multicolumn{3}{|l|}{ Site of infection } \\
\hline CVC & 1 & \\
\hline Catheter-associated UTI & 4.16 (1.86 to 9.33$)$ & 0.001 \\
\hline Non-catheter-associated UTI & $2.93(1.20$ to 7.14$)$ & 0.018 \\
\hline Biliary tract & 0.78 (0.09 to 6.65$)$ & 0.821 \\
\hline Other & 2.34 (1.17 to 4.70$)$ & 0.016 \\
\hline \multicolumn{3}{|c|}{ Delay in appropriate treatment (days) } \\
\hline$<1$ & 1 & \\
\hline$\geq 1$ and $<2$ & 1.11 (0.63 to 1.98$)$ & 0.717 \\
\hline$\geq 2$ and $<3$ & $0.67(0.27$ to 1.68$)$ & 0.394 \\
\hline$\geq 3$ & $1.24(0.40$ to 3.81$)$ & 0.712 \\
\hline \multicolumn{3}{|l|}{ Organism susceptibility } \\
\hline Fully susceptible & 1 & \\
\hline Partially resistant & $1.11(0.67$ to 1.81$)$ & 0.690 \\
\hline Multiple drug resistance & $1.54(0.94$ to 2.54$)$ & 0.090 \\
\hline
\end{tabular}

Table 3 Multivariate logistic regression analysis of mortality at 7-days

\begin{tabular}{lll}
\hline & Adjusted OR(95\% CI) & p Value \\
\hline Age (years) & & \\
$<31$ & 1 & \\
$31-50$ & $3.64(0.26$ to 10.46$)$ & 0.599 \\
$51-70$ & $5.37(1.15$ to 25.05$)$ & 0.133 \\
$>70$ & & 0.033 \\
CCI & 1 & \\
0 & $0.99(0.32$ to 3.06$)$ & 0.992 \\
1 & $1.70(0.59$ to 4.91$)$ & 0.331 \\
2 & $1.96(0.67$ to 5.72$)$ & 0.217 \\
3 & $2.36(0.75$ to 7.41$)$ & 0.140 \\
4 & $1.67(0.45$ to 6.14$)$ & 0.440 \\
5 & $2.94(0.92$ to 9.44$)$ & 0.069 \\
6 & $3.41(1.09$ to 10.66$)$ & 0.035 \\
$7-9$ & & 0.019 \\
Site of infection & 1 & 0.0519 \\
CVC & $2.90(1.19$ to 7.07$)$ & \\
Catheter-associated UTI & $2.12(0.82$ to 5.44$)$ & \\
Non-catheter-associated UTI & $0.44(0.05$ to 3.97$)$ & \\
Biliary tract & $2.11(1.00$ to 4.47$)$ & \\
Other & & \\
\hline Generalised estimating equations were used to account for dependency between \\
multiple bacteraemic episodes for 84 patients.
\end{tabular}

bacteraemic episodes were available for inclusion. In total, three patients with incomplete CCI data were excluded. After adjustment for age and CCI, the association between catheterassociated UTIs and death at 7 days remained significant (OR $2.90,95 \%$ CI 1.19 to 7.07$)$ although, at 30 days, this association became non-significant (OR 1.32, 95\% CI 0.67 to 2.61).

\section{DISCUSSION}

This is the first UK-based study, for more than two decades, to benchmark mortality in consecutive patients with hospitalacquired bacteraemia. Age, CCI, Pitt bacteraemia score and site of infection were significantly associated with 7-day mortality although, uniquely, compared with CVC-associated bacteraemic infection, we demonstrated a significant association between catheter-associated bacteraemic UTIs and death at 7 days. Seven-day mortality associated with catheter-associated bacteraemic UTIs was significantly higher than CVC-associated bacteraemic infection, even after adjustment for age and comorbidities, recognised confounders in other studies. ${ }^{9}$ In our multivariate logistic regression analysis, we did not adjust for Pitt score, a marker of severity, as this is on the causal pathway. The causal pathway, we hypothesised, was that urinary catheterisation can lead to bacteriuria and severe bacteraemic infections resulting in death at 7 days. Adjustment for Pitt scores would not have enabled our a priori hypothesis to be tested.

Although asymptomatic bacteriuria occurs commonly in catheterised patients and does not require treatment unless the patient becomes symptomatic, ${ }^{16}$ bacteriuria is a prerequisite for septicaemia. Urinary catheterisation is also an independent predictor for bacteraemic UTIs. ${ }^{17}$ Although outcome studies of hospital and healthcare-associated bacteraemia emphasise the contribution of Gram-negative infections, ${ }^{18}$ only two emphasise the contribution of catheter-associated UTIs. ${ }^{19}{ }^{20}$ One of these 
studies ${ }^{20}$ benchmarked 30 -day mortality at $30.8 \%$ but attributed death to $12.8 \%$, a proportion significantly lower than our 7-day mortality (30.1\%). Our observation was that most patients who died from catheter-associated UTIs died early, within 7 days, and that most deaths were likely to be caused by infection. We did not observe a substantial rise in deaths between 7 and 30 days, many of which may have been due to other causes. It is possible that some of these deaths were preventable. One study demonstrated a $20 \%$ reduction in catheter-associated UTIs following better patient selection and adherence to best practice. ${ }^{21}$

$E$ coli is the predominant organism isolated from catheterised patients with bacteriuria ${ }^{22}$ and bacteraemia ${ }^{23}$ but a variety of other organisms also cause infection. In our cohort, cultures from a catheterised specimen of urine (CSU) most commonly yielded mixed organisms including coliforms. This is because it is not routine laboratory practice to speciate and perform susceptibility testing on bacterial isolates from a CSU unless there is a pure bacterial growth. Unfortunately, some CSU samples were not sent for processing and other CSU samples demonstrated pyuria but no bacterial growth, probably secondary to antimicrobial administration prior to sample collection. A smaller number of CSU samples grew an isolate different from the bacteraemic isolate. Although some of these may have been misidentified on chromogenic agar, possibly within a mixed culture, we cannot be sure that these bacteraemias were truly caused by catheterassociated UTIs.

Overall, medical patients had a significantly higher 7-day mortality compared with patients in other specialities. A likely explanation is that these patients were more elderly and had more comorbidities. Consequently, some of these patients would have been unsuitable for critical care transfer and others with terminal malignancy would have been placed on a palliative care pathway. We did not quantify these groups of patients.

There were other limitations to this study. We did not include patients with healthcare-associated infections, which we considered to be a subset of community-acquired infections. Like all bacteraemia studies, septic patients who were not cultured were excluded. This may have led to an underestimate in the number of episodes of bacteraemia and the number of deaths caused by septicaemia. Time from onset of sepsis to treatment was not recorded and we did not take into account time taken to switch from empirical to definitive treatment. A large proportion of patients had undefined sites of infection, many who died early

\section{Main messages}

- Hospital-acquired bacteraemia is a significant cause of patient mortality and morbidity. Among consecutive patients with hospital-acquired bacteraemia, 7-day mortality was $90 /$ $437(20.6 \%)$.

- Compared with central venous catheter-associated bacteraemic infections, catheter-associated bacteraemic urinary tract infections (UTIS) were significantly associated with 7-day mortality. This association remained significant after adjustment for age and comorbidities.

- Because the Department of Health used meticillin-resistant Staphylococcus aureus bacteraemia as a key quality indicator, attention is focused upon reducing intravascular catheter-associated infections in most UK NHS trusts. Based on our findings, we advocate a shift of emphasis towards reducing catheter-associated UTIs in hospitalised patients.

\section{Current research questions}

- Should 7-day outcomes for hospital-acquired bacteraemia, stratified for age, Pitt score and Charlson comorbidity index be used as a quality indicator, enabling comparisons across different NHS trusts?

- Will mandatory Escherichia coli bacteraemia surveillance result in a reduction in catheter-associated urinary tract infections in hospitalised patients?

- Might hospital-acquired bacteraemia surveillance, focusing on infection caused by medical devices, be a better way of reducing all bacteraemic hospital-acquired infections?

before the site of infection could be established. If a significant number were caused by catheter-associated UTIs, this may have biased the results. Although adjustments for age and CCI (a composite score for comorbidities including renal failure) were made, we did not adjust for underlying urological anatomical abnormalities. Also, after checking our trust's computerised patient administration system for re-admissions, we assumed that once patients recovered from infection or were discharged home, they survived until 7 days. However, in sensitivity analysis, where we assumed discharged patients died within 7 days, our findings remained the same. We determined all-cause mortality and did not determine death to be directly attributable to infection, although, like others, ${ }^{24}$ we believe that death within 7 days is likely to have been secondary to infection. Due to incomplete data, only three patients were excluded from multivariate regression analysis.

In 2011, the Department of Health (England) launched mandatory $E$ coli bacteraemia surveillance highlighting the need for more data on Gram-negative bacteraemias and their sources. Rather than E coli surveillance, we have previously advocated a

\section{Key references}

- National Nosocomial Infections Surveillance (NNIS). System Report, data summary from January 1992 through June 2004, issued October 2004. Am J Infect Control 2004;32:470-85.

- Coello R, Charlett A, Ward V, et al. Device related sources of bacteraemia in English hospitals. Opportunities for the prevention of hospital-acquired bacteraemia. J Hosp Infect 2003;53(1):46-57.

- Jain P, Parada JP, David A et al. Overuse of the indwelling urinary tract catheter in hospitalised medical patients. Arch Inter Med 1995;155:1425-29.

- Hooton TM, Bradleys F, Cordenas DD, et al. Diagnosis, prevention and treatment of catheter-associated urinary tract infection in adults: 2009 International Clinical Practice Guidelines from the Infectious Diseases Society of America. Clin Infect Dis 2010;50:625-63.

- Knoll BM, Wright D, Ellingson L, et al. Reduction of inappropriate urinary catheter use at a Veterans Affairs hospital through a multifaceted quality improvement programme. Clin Infect Dis 2011;52:1283-90.

- Melzer M, Welch C. Is Escherichia coli bacteraemia preventable? Lancet Infect Dis 2012;12:103-4. 
nationwide extended bacteraemia surveillance programme, focusing on medical devices such as urinary catheters, as a better way of reducing hospital-acquired bacteraemic UTIs. ${ }^{25}$ Success in managing catheter-associated UTIs depends upon prompt recognition, early administration of appropriate antibiotics, removing or changing a catheter and patient management in an appropriate environment depending upon severity of infection. There is a need to educate healthcare workers to avoid routine use of urinary catheters, to remove them when they are no longer needed and to use alternative methods for care of incontinence. In comparison to CVC-associated bacteraemic infection, catheter-associated bacteraemic UTIs are significantly associated with 7-day mortality and efforts to reduce these infections should be prioritised.

Acknowledgements We acknowledge four other Consultant Microbiology colleagues, Sandra Lacey, Lindsey Bain, Simon Namnyak and Artemio Yaneza who helped in collecting these data.

Contributors $\mathrm{MM}$ had the original idea and sort to prove his hypothesis by interrogating the BHRUT bacteraemia data set. CW helped analyse the data.

Competing interests None.

Ethics approval BHRUT clinical governance group.

Provenance and peer review Not commissioned; externally peer reviewed.

Open Access This is an Open Access article distributed in accordance with the Creative Commons Attribution Non Commercial (CC BY-NC 3.0) license, which permits others to distribute, remix, adapt, build upon this work non-commercially, and license their derivative works on different terms, provided the original work is properly cited and the use is non-commercial. See: http://creativecommons.org/licenses/by-nc/3.0/

\section{REFERENCES}

1 National Nosocomial Infections Surveillance (NNIS). System Report, data summary from January 1992 through June 2004, issued October 2004. Am J Infect Control 2004;32:470-85.

2 Coello R, Charlett A, Ward V, et al. Device related sources of bacteraemia in English hospitals. Opportunities for the prevention of hospital-acquired bacteraemia. J Hosp Infect 2003;53:46-57

3 Jain $\mathrm{P}$, Parada JP, David A, et al. Overuse of the indwelling urinary tract catheter in hospitalised medical patients. Arch Inter Med 1995;155:1425-29.

4 Gardam MA, Amihod B, Orenstein P, et al. Overutilization of indwelling urinary catheters and the development of nosocomial urinary tract infections. Clin Perform Qual Health Care 1998;6:99-102.

5 Wald $\mathrm{HL}$, Epstein AM, Radcliff TA, et al. Extended use of urinary catheters in older surgical patients: a patient safety problem? Infect Control Hosp Epidemiol 2008;29:116-24.
6 Loeb M, Hunt D, O'Halloran K, et al. Stop orders to reduce inappropriate urinary catheterizations in hospitalized patients: a randomized controlled trial. J Gen Intern Med 2008;23:816-20.

7 Platt R, Polk BF, Murdock B, et al. Reduction in mortality associated with nosocomial urinary tract infection. Lancet 1983;1:893-7.

8 Saint S, Lipsky BA. Preventing catheter-related bacteriuria: should we? Can we? How? Arch Intern Med 1999:159:800-8.

9 Hooton TM, Bradleys F, Cordenas DD, et al. Diagnosis, prevention and treatment of catheter-associated urinary tract infection in adults: 2009 International Clinical Practice Guidelines from the Infectious Diseases Society of America. Clin Infect Dis 2010;50:625-63.

10 Garner JS, Jarvis WR, Emori TG, et al. CDC definitions for nosocomial infections, 1988. Am J Infect Control 1988;16:128-40.

11 McGregor JC, Rich SE, Harris AD, et al. A systematic review of the methods used to assess the association between appropriate antibiotic therapy and mortality in bacteraemic patients. Clin Infect Dis 2007:45:329-37.

12 Dellinger RP, Carlet JM, Masur $\mathrm{H}$, et al. Surviving sepsis campaign guidelines for management of severe sepsis and septic shock. Intensive Care Med 2004;30:536-55.

13 Charlson ME, Pompei $\mathrm{P}$, Ales $\mathrm{KL}$, et al. A new method of classifying prognostic comorbidity in longitudinal studies: development and validation. J Chronic Dis 1987;40:373-83.

14 Paterson DL, Ween-Chien Ko, Gottberg AV, et al. International prospective study of Klebsiella pneumoniae bacteraemia: implications of extended spectrum beta-lactamase production in nosocomial infections. Ann Intern Med 2004;140:26-32.

15 Andrew JM. BSAC standardised disc susceptibility testing method (version 6). J Antimicrob Chemother 2007:60:20-41.

16 Tambyah PA, Maki DG. Catheter associated urinary tract infection is rarely symptomatic. A prospective study of 1497 catheterized patients. Arch Intern Med 2000;106:678-82.

17 Bahagan $Y$, Raveh $D$, Schlesinger $Y$, et al. Prevalence and predictive features of bacteraemic UTI in emergency dependent patients. Eur J Clin Microbiol Infect Dis 2007;26:349-52.

18 Edgeworth JD, Treacher DF, Eykyn SJ. A 25-year study of nosocomial bacteraemia in an adult intensive care unit. Crit Care Med 1999:27:1421-8.

19 Rudman D, Hontanosas A, Cohen Z, et al. Clinical correlates of bacteraemia in a Veterans Administration extended care facility. J Am Geriatr Soc 1988;36:726-32

20 Bryan CS, Reynolds KL. Hospital-acquired bacteraemic urinary tract infection: epidemiology and outcome. J Urol 1984;132:494-8.

21 Knoll BM, Wright D, Ellingson L, et al. Reduction of inappropriate urinary cathete use at a Veterans Affairs hospital through a multifaceted quality improvement programme. Clin Infect Dis 2011;52:1283-90.

22 Nicolle LE. Catheter-related urinary tract infection. Drugs Aging 2005:22:627-39.

23 Mylotte JM. Nursing home-acquired bloodstream infection. Infect Control Hosp Epidemiol 2005:26:833-7.

24 Haug JB, Harthug S, Kalager T, et al. Bloodstream infections at a Norwegian University Hospital, 1974-79 and 1988-89: changing etiology, clinical features and outcomes. Clin Infect Dis 1994;19:246-56.

25 Melzer M, Welch C. Is Escherichia coli bacteraemia preventable? Lancet Infect Dis 2012;12:103-4. 